\title{
Acute ischemic preconditioning does not influence high- intensity intermittent exercise performance
}

\author{
Isabela Coelho Marocolo ${ }^{1}$, Gustavo Ribeiro da Mota ${ }^{\text {Corresp., }}{ }^{1}$, André Monteiro Londe ${ }^{1}$, Stephen D Patterson ${ }^{2}$, \\ Octávio Barbosa Neto ${ }^{1}$, Moacir Marocolo ${ }^{3}$ \\ ${ }^{1}$ Human Performance and Sport Research Group, Department of Sport Sciences, Institute of Health Sciences, Federal University of Triangulo Mineiro, \\ Uberaba, Brazil, Uberaba, MG, Brazil \\ 2 School of Sport, Health, and Applied Science, St. Mary's University, Twickenham, UK \\ 3 Physiology and Human Performance Research Group, Department of Physiology, Institute of Biological Sciences, Federal University of Juiz de Fora, Juiz \\ de Fora, MG, Brazil \\ Corresponding Author: Gustavo Ribeiro da Mota \\ Email address: grmotta@gmail.com
}

This study evaluated the acute effect of ischemic preconditioning (IPC) on a high-intensity intermittent exercise performance and physiological indicators in amateur soccer players. Thirteen players ( $21.5 \pm 2$ yrs) attended 3 trials separated by 3-5 days in a counterbalanced randomized cross-over design: IPC (4x5-min occlusion 220 $\mathrm{mmHg} /$ reperfusion $0 \mathrm{mmHg}$ ) in each thigh; SHAM (similar to the IPC protocol but "occlusion" at $20 \mathrm{mmHg}$ ) and control (seated during the same time of IPC). After 6-min of each trial (IPC, SHAM or control), the players performed the YoYo Intermittent Endurance Test level 2 (YoYolE2). The distance covered in the YoYolE2 (IPC $867 \pm 205$ m; SHAM 873 $\pm 212 \mathrm{~m}$; control $921 \pm 206 \mathrm{~m}$ ) was not different among trials $(p=0.10)$, furthermore, lactate concentration and rate of perceived exertion did not differ $(P>0.05)$ among protocols. There were also no significant differences in either mean heart rate (HR) or peak HR ( $p>0.05$ ) for both IPC and SHAM compared to control. Therefore, we conclude that acute IPC does not influence high-intensity intermittent exercise performance in amateur soccer players and that rate of perceived exertion, heart rate and lactate do not differ between the intervention IPC, SHAM and control. 
1 Acute ischemic preconditioning does not influence high-intensity intermittent exercise 2 performance

3

4 Short Title: Ischemic preconditioning and yoyo test

5

6 Research Article

7

8 Isabela Coelho Marocolo ${ }^{1}$, Gustavo Ribeiro da Mota ${ }^{1, *}$, André Monteiro Londe ${ }^{1}$, Stephen D.

9 Patterson $^{2}$, Octávio Barbosa Neto ${ }^{1}$, Moacir Marocolo ${ }^{3}$.

${ }^{1}$ Human Performance and Sport Research Group, Department of Sport Sciences, Institute of Health Sciences, Federal University of Triangulo Mineiro - UFTM/Brazil - Av. Tutunas 490 Uberaba/MG - Brazil, 38061-500

${ }^{2}$ School of Sport, Health, and Applied Science, St. Mary's University, UK.

${ }^{3}$ Physiology and Human Performance Research Group, Department of Physiology, Institute of Biological Sciences, Federal University of Juiz de Fora, UFJF/Brazil - Rua José Lourenço Kelmer s/n - Juiz de Fora/MG - Brazil, 36036-900

* Corresponding author:

grmotta@gmail.com - Gustavo Ribeiro da Mota - Human Performance and Sport Research Group, Department of Sport Sciences/Institute of Health Sciences, Federal University of Triângulo Mineiro, Uberaba, Brazil.

Av. Tutunas, 490 Uberaba/MG - 38061-500. Phone: 5534 3700-6633 


\section{Abstract}

27 This study evaluated the acute effect of ischemic preconditioning (IPC) on a high-intensity 28 intermittent exercise performance and physiological indicators in amateur soccer players. 29 Thirteen players (21.5 \pm 2 yrs) attended 3 trials separated by 3-5 days in a counterbalanced 30 randomized cross-over design: IPC (4x5-min occlusion $220 \mathrm{mmHg} /$ reperfusion $0 \mathrm{mmHg}$ ) in each 31 thigh; SHAM (similar to the IPC protocol but "occlusion" at $20 \mathrm{mmHg}$ ) and control (seated 32 during the same time of IPC). After 6-min of each trial (IPC, SHAM or control), the players performed the YoYo Intermittent Endurance Test level 2 (YoYoIE2). The distance covered in the YoYoIE2 (IPC $867 \pm 205 \mathrm{~m}$; SHAM $873 \pm 212 \mathrm{~m}$; control $921 \pm 206 \mathrm{~m}$ ) was not different among trials $(\mathrm{p}=0.10)$, furthermore, lactate concentration and rate of perceived exertion did not differ $(P>0.05)$ among protocols. There were also no significant differences in either mean heart rate $(\mathrm{HR})$ or peak HR $(\mathrm{p}>0.05)$ for both IPC and SHAM compared to control. Therefore, we conclude that acute IPC does not influence high-intensity intermittent exercise performance in amateur soccer players and that rate of perceived exertion, heart rate and lactate do not differ between the intervention IPC, SHAM and control.

Key-words: blood occlusion, physical training, ischemia, skeletal muscle, ergogenic. 
47

48

49

50

51

52

\section{Introduction}

The technique of ischemic preconditioning (IPC) alternates brief periods of occlusion and reestablishment (e.g., 3 to 5-min each) of muscle blood flow prior to exercise using a tourniquet applied in the proximal part of a limb (e.g., lower limbs) (Marocolo et al. 2016a) aiming to improve exercise performance. The ergogenic effects are still debatable (Marocolo et al. 2016a) especially because some study designs are limited (e.g., without placebo-control) (da Mota \& Marocolo 2016) and the mechanism behind IPC as well as its supposed exercise enhancement has yet to be clarified. Potential mechanisms of IPC exercise enhancement are hyperemia (5-6 fold increase in muscle blood flow) during reperfusion (Libonati et al. 2001), attenuated ATP depletion, and increased phosphocreatine production/oxygen uptake during the reperfusion phase (Andreas et al. 2011). Additionally, IPC has been suggested to improve aerobic and anaerobic performance (Cruz et al. 2015; Incognito et al. 2016) mostly in high-intensity exercise in cyclic modalities (e.g., running, cycling, swimming).

Although one study (Gibson et al. 2015) evaluated the IPC effect in athletes with a history of participation in team sport and others in repeated sprint cycling (Patterson et al. 2015), no study has examined its effects on high-intensity intermittent exercise (i.e., specific to team sports). The YoYo intermittent endurance test level 2 (YoYoIE2) simulates high-intensity intermittent exercise similar to the activity seen in soccer, and is effective due to its simplicity and reproducibility (Bradley et al. 2011), and it is correlated with both oxygen uptake and citrate synthase activity in untrained men (Krustrup et al. 2015). Although Krustrup et al. (2015) stated that aerobic energy production plays a "more important role" for fatigue resistance in untrained subjects versus trained male soccer players during the YoYoIE2, their results showed also an important contribution of the glycolytic pathway in the YoYoIE2 (e.g. blood lactate $11 \mathrm{mmol}$ at 5-min of recovery). Since IPC seems to improve performance in exercise tests of predominantly lactic anaerobic and aerobic capacity (Incognito et al. 2016), the aim of this study was to evaluate the acute effect of IPC on YoYoIE2 performance and some physiological parameters in amateur soccer players. We hypothesized that IPC would increase the distance covered in YoYoIE2, because this test for our specific sample (amateur players) would be suitable to recruit predominantly both the glycolytic and aerobic systems and, therefore, meet the ergogenic potential from IPC (Cruz et al. 2015; Incognito et al. 2016). 


\section{Materials and Methods}

Thirteen male amateur soccer players $(21.5 \pm 2 \mathrm{yrs}, 173 \pm 5 \mathrm{~cm}, 69.7 \pm 6 \mathrm{~kg}, 12.6 \pm 5$ body fat \%) volunteered for this study. The players participated in $2.7 \pm 0.5 \mathrm{~h} . \mathrm{wk}^{-1}$ of training and $1.3 \pm$ 0.2 matches per week and playing experience of $11 \pm 4$ yrs. Inclusion criteria were: a) soccer playing experience more than 5 yrs, b) no smoking history during the last year, c) absence of any cardiovascular or metabolic disease, d) systemic blood pressure lower than 140/90 $\mathrm{mmHg}$ and no use of antihypertensive medication, e) no use of creatine supplementation, anabolic steroids, drugs or medication with potential effects on physical performance (self-reported) and g) no recent musculoskeletal injury. This study was approved by the local institutional Ethical Committee for Human Experiments (Federal University of Triangulo Mineiro - 993.636/2015) and was performed in accordance with ethical standards in sports science research (Harriss \& Atkinson 2015). In addition, all subjects signed an informed consent form. Based on prior research (Bradley et al. 2011), a sample size less than 13 (i.e., n between 6 and 9) was sufficient to detect a significant $(\mathrm{p}<0.05)$ difference among playing positions ("group") in the YoYoIE2 (main dependent variable). Also, a study using a similar IPC protocol (independent variable) and physical test duration, reported significant statistical effects with a sample size of 12 individuals (Cruz et al. 2015). Thus, to counteract any potential drop out, a sample of 13 subjects was included for this study.

\section{Experimental design of the study}

Subjects attended the laboratory five times (with 3 to 5 days in-between), for initial screening and anthropometric measurements and for familiarization with the equipment and the test. In the $3^{\text {rd }}, 4^{\text {th }}$ and $5^{\text {th }}$ visits, a randomized crossover assignment (IPC, SHAM and control) was adopted and the YoYoIE2 was carried out after 6-min of each trial (IPC, SHAM or control). All YoYoIE2 tests were conducted by the same experienced researcher in a constant environment $\left(23 \pm 2^{\circ} \mathrm{C}\right.$; Humidity: $75 \pm 4 \%$ ) at the same time of the day $(9: 00-11: 00 \mathrm{~h})$. To prevent the possibility of a placebo (positive) effect (Marocolo et al. 2017; Marocolo et al. 2016b; Marocolo et al. 2016c), all subjects were informed that all conditions could improve performance. Also, in order to prevent nocebo (negative) effects the subjects were informed that IPC and SHAM would cause absolutely no harm, despite discomfort related to the maneuvers (Ferreira et al. 2016). 
109 Additionally, the tester was blinded for which protocol (i.e. IPC, SHAM or control) the subjects

110 had undergone before. Also the subjects were kept blinded in relation to performance and other

111 indicators until the end of the research, i.e., no information about distance covered (the audio of

112 speed and level of YoYoIE2 was in unknown language), HR and lactate. Coffee (or caffeine

113 products), tea, and alcohol intake was prohibited as well as strenuous exercise for 48 hours

114 before testing (Incognito et al. 2016).

115

116 Ischemic preconditioning protocol

117 The IPC maneuver consisted of four cycles of 5-min occlusion (220 $\mathrm{mmHg})$ and 5-min 118 reperfusion (no pressure) in each thigh (total duration 40-min), using a pneumatic tourniquet

119 (komprimeter Riester ${ }^{\circledR}$, Jungingen, Germany) administered at the subinguinal region of the 120 thighs. We applied this specific IPC protocol for the following reasons: a) several studies have 121 successfully explored the ergogenic effects of IPC on exercise performance with the same 122 protocol (Cruz et al. 2015; Jean-St-Michel et al. 2011; Marocolo et al. 2016a; Patterson et al. 123 2015); b) at least three ischemia-reperfusion cycles are necessary to protect against skeletal 124 muscle infarction and endothelial dysfunction after prolonged periods of imposed ischemia 125 (Pang et al. 1995) and c) it is considered safe and well tolerated in both patients and healthy 126 volunteers (Gonzalez et al. 2014). The occlusion and reperfusion phases were conducted 127 alternately between the thighs, with subjects remaining seated (knee at $90^{\circ}$ angle). The 128 effectiveness of occlusion in the IPC session was controlled by auscultation of the arteries 129 around the ankle (Loenneke et al. 2012). In the SHAM protocol, an external pressure of 20 $130 \mathrm{mmHg}$ was administered, as proposed in previous studies (Foster et al. 2011; Marocolo et al. 131 2016a). For the control condition, the subjects remained seated for a respective period of time. 132 Although a consensus does not exist with regards to the time between the last IPC cycle and the 133 exercise testing, ranging from 5-min to 90-min in most studies with exercise performance 134 (Marocolo et al. 2016a), the IPC has been shown to improve exercise performance within 45-min 135 of the final cuff inflation (Bailey et al. 2012; Patterson et al. 2015). Thus, in the current study, 136 the warm-up to the YoYoIE2 was performed after 6-min of each trial (i.e. IPC, SHAM or 137 control). 
140 Before each session, all subjects indicated a score on a perceived recovery scale (Laurent et al. 141 2011), (from 0 "very little recovered, feeling extremely tired" to 10 "very well recovered, feeling 142 with great energy"), about their relative physical recovery to make sure the player was in the 143 same recovery condition before the trials. If a player scored 4 or less (somewhat recovered) for 144 his recovery status, he was excluded from that day's session and invited to wait until the next day 145 to perform the session.

146

147

Yo-Yo Intermittent Endurance Test Level 2 (YoYoIE2):

148

149

150

151

152

153

154

155

156

157

158

159

160

161

162

163

164

165

166

167

168

169

After 6-min of recovery from each trial (IPC, SHAM or control) the warm-up for the YoYoIE2 began. The warm-up consisted of performing the first three running bouts of the YoYoIE2 followed by a period of lower-extremity stretching. All players were familiar with the YoYoIE2 because they used this test on regular basis for fitness evaluation purpose. The YoYoIE2 consists of a repeated 2 × 20-m shuttle run at progressively increasing speed stages (initial speed $\sim 12$ $\mathrm{km} . \mathrm{h}-1$ ), guided by specific audio (5-s to recovery in a marked $2.5 \times 2 \mathrm{~m}$ area behind the finishing line). Cessation of the test was assessed by failure to reach the finish line by the tone on two occasions(Bradley et al. 2011). The YoYoIE2 is a reproducible, sensitive tool associated with soccer match performance and can differentiate intermittent exercise performance of several standards and is therefore highly suggested for soccer players (Bradley et al. 2014; Bradley et al. 2012). For consistency, the subjects performed the YoYoIE2 in groups of three or four (always the same group) and they received similar verbal encouragement during the tests.

\section{Heart rate (HR), rate of perceived exertion (RPE) and blood lactate concentration:}

HR was monitored throughout the entire YoYoIE2, by an individual RS800CX heart monitor (Polar Electro ${ }^{\circledR}$, Kempele, Finland). After the YoYoIE2, the player indicated (individually to prevent influence from other player) a score for his RPE via CR-10 Borg scale in order to determine the subjective intensity of the session. This scale ranges from 0 to 10 , where 0 is "nothing at all" and 10 is "very very hard (maximal)" (Borg 2007; Borg \& Kaijser 2006). Blood samples $(25 \mu \mathrm{L})$ were collected from the fingertip 4-min post YoYoIE2 using a lancet, and the lactate concentration was measured via a valid (Fell et al. 1998) portable lactate analyzer (Accutrend ${ }^{\circledR}$ Plus system, ROCHE, Basel Switzerland). 
171 Statistical analysis

172 The Shapiro-Wilk test was applied to verify the normal distribution of the data. For between173 protocol analysis, one-way analysis of variance (ANOVA) for repeated measures was conducted, 174 followed by post-hoc Tukey's test or nonparametric ANOVA (Friedman test) followed by a 175 post-hoc Dunn's test was performed. Only for the distances covered in the YoYoIE2 we 176 calculated the effect size (ES; Cohen d) to determine the meaningfulness of the difference 177 (practical relevance), classified as: trivial $(<0.2)$, small $(>0.2-0.6)$, moderate $(>0.6-1.2)$, large $(>$ 178 1.2-2.0) and very large (>2.0) as recommended (Batterham \& Hopkins 2006). The significance 179 level was set at 0.05. The software used for data analysis was GraphPad ${ }^{\circledR}$ (Prism 6.0, San Diego, 180 CA, USA).

181

\section{Results}

183 184 185 186

Before the trials, the perceived recovery scores were not different $(P=0.1)$ among the protocols $(\mathrm{IPC}=6.9 \pm 1.7, \mathrm{SHAM}=7.7 \pm 1.5$ and control $=7.7 \pm 0.7)$. RPE and lactate concentrations did not differ after the trial among all protocols. Mean HR and peak HR presented no difference $(\mathrm{p}=$ 0.17 and $p=0.06$, respectively) among the three trials (Table 1 ).

\section{Table 1 near here}

The distance covered in the YoYoIE2 did not differ $(P=0.10 ; \mathrm{ES}=0.16)$ among the protocols (Figure 1). The mean distances were: IPC $867 \mathrm{~m}(S D=205)$, SHAM $873 \mathrm{~m}(S D=212)$ and control $921 \mathrm{~m}(S D=206)$. The effect sizes were 0.26 (small) IPC vs control, 0.2 (trivial) SHAM vs control and 0.03 (trivial) IPC vs SHAM.

Figure 1 near here

Figure 2 shows the individual change in distances covered by each player in the YoYoIE2. It should be noted that only three players ( $23 \%)$ performed better after SHAM and four ( $\sim 30 \%)$ after IPC, while the others virtually covered the same distances or even shorter distances than under control conditions.

\section{Figure 2 near here}




\section{Discussion}

200

201

202

203

204

205

206

207

208

209

210

211

212

213

214

215

216

217

218

219

220

221

222

223

224

225

226

227

The purpose of this study was to evaluate the acute effect of IPC on YoYoIE2 performance and related physiological indicators in amateur soccer players. Our main findings are that acute IPC did not influence performance in the YoYoIE2. In addition, IPC did not influence perceived exertion, blood lactate concentrations (after the test) or either mean or peak HR in comparison with SHAM and control interventions.

Our current study is the first to evaluate the IPC effect on a high-intermittent performance test that involves changing of direction, and acceleration/deceleration (YoYoIE2), all of which is important for team sports, specifically soccer (Bradley et al. 2011; Paul et al. 2016; Varley \& Aughey 2013). The distance covered in the YoYoIE2 did not differ significantly among the three conditions, showing no influence (i.e., neither positive nor negative), as well as no practical relevance (i.e.; trivial effect sizes) of acute IPC on this sort of test and population. The distance covered in the current study in the control conditions $(935 \pm 232 \mathrm{~m})$ is higher than that reported for untrained men $(665 \pm 271 \mathrm{~m})$ but lower than highly trained soccer players $(2027 \pm 298 \mathrm{~m})$ (Krustrup et al. 2015). Since the scores of perception of recovery were similar before all of the three trials (i.e., players starting under same conditions), basic conditions for comparison of different protocols were ensured.

According to a recent systematic review (Incognito et al. 2016), the most consistent benefit of IPC would be an improvement in exercise tests with the prevalence of energy coming from glycolytic and aerobic sources. The YoYoIE2 has been designed to stimulate both anaerobic and aerobic pathways; however despite this, IPC had no effect on intermittent exercise lasting 6min. These same authors have argued that there are IPC responders (i.e. improvements in performance) and non-responders (i.e., no effects on performance) phenotypes, due to large between-subject variability of results and, therefore, they recommend prudence in the interpretation of mean group changes in exercise performance due to IPC (Incognito et al. 2016). In other words, individual analysis should also be performed to more thoroughly assess the potential ergogenic effects of IPC on exercise performance. Analyzing the individual responses 
228 of YoYoIE2 in the current study, we found only three and four players better in SHAM and IPC

229 protocols, respectively. These results suggest that acute IPC does not enhance high-intensity 230 intermittent exercise performance for the majority of the players. Considering that the same acute

231 IPC protocol (i.e., 4 X 5 cycles of occlusion/reperfusion) improved endurance performance $232(\sim 8 \%)$ with exercise time duration very close to ours ( $\sim 6-\mathrm{min})$ in recreational cyclists (Cruz et al. 233 2015), we may speculate that the nature of YoYoIE2; i.e., intermittent maximal test, including 234 acceleration and deceleration, changing of directions, important eccentric actions involved, 235 might counterbalance the effect following acute IPC. Future studies should explore this 236 hypothesis more deeply by including other kinds of YoYo test (e.g., recovery level I).

237

238 Recent evidence suggests the possibility of a placebo (positive) or nocebo (negative) effect 239 (Ferreira et al. 2016; Marocolo et al. 2015), but in the current study, this potential bias was 240 controlled by three methods: first, all subjects were informed that both maneuvers could improve performance (SHAM and IPC) and none of them could harm performance; second, we kept the tester blinded in relation to the previous protocol (i.e., the physical tester did not know if the player had performed IPC, SHAM or control) and third, no information about distance covered, HR and lactate were provided.

The RPE is used in studies as an essential and valid indicator of exercise intensity, in addition to the measurement of traditional physiological variables (Borg \& Kaijser 2006). In our study, RPE was checked immediately after each test and reached high values, suggesting near maximal internal intensity in the tests. However, RPE did not differ in any condition. Considering that IPC effects could have any motivational basis on exercise performance (Marocolo et al. 2015; Marocolo et al. 2016b; Marocolo et al. 2016c) the register of the RPE for evaluating psychophysical parameters would assist in intensity measures. Our current results showed that IPC did not affect the RPE and it is consistent with previous studies: after sprints series compared to SHAM (Gibson et al. 2015) and after a five-kilometer race to the control intervention (Bailey et al. 2012).

Heart rate was measured continuously during the test, and neither peak nor mean HR differed 258 among interventions (IPC, SHAM and CON). One study showed a shortening QT interval during 
259 exercise, without any RR interval changes after IPC intervention (Caru et al. 2016). Conversely, 260 in another study (Clevidence et al. 2012) significantly higher values for the same parameters in 261 cyclists were found after IPC intervention. For these authors, the HR increase could be explained

262 by a decrease in compensation to a reduced stroke volume and therefore cardiac output at the 263 time of the IPC maneuver, as previously described (Iida et al. 2007). However, our values of 264 covered distance were not different among interventions; nine and eight subjects covered a little 265 shorter distance after SHAM and IPC, respectively, compared to control, which could explain, at 266 first glance, no difference in values of peak and mean HR during the YoYoIE2 following IPC 267 and SHAM interventions.

268

269 In our study, blood lactate concentrations are in line with the literature for YoYoIE2 (Krustrup et 270 al. 2015) in similar subjects (i.e., untrained) confirming also the significant anaerobic 271 contribution to ATP supply. Another study showed that IPC generated lower blood lactate 272 concentration at submaximal intensity during an incremental running test (Bailey et al. 2012), 273 but in the current study we did not find any difference among IPC, SHAM and control conditions. This difference is probably due to the type of exercise, since in the present study we applied an intermittent maximal test.

276

277 There are several limitations to the present study: the absence of measurements to understand the mechanisms underpinning the IPC/SHAM maneuvers, as well the YoYoIE2, such as muscle 279 blood flow, muscle oxy/deoxyhemoglobin, oxygen uptake, enzyme activities, serum nitric oxide, and partial oxygen/carbon dioxide pressures. Also, considering that reactive oxygen species play an important role in both IPC (Zhu \& Zuo 2013) and exercise (He et al. 2016), measurements of reactive oxygen species for both IPC and exercise test would be interesting. Our sample size (n =13) was relatively small, and it may generate a type II error. However, it is higher than one study that applied the same IPC protocol and found statistically significant effects from IPC in cyclists performing similar duration of maximal exercise (Cruz et al. 2015). Lastly, despite our control to avoid potential placebo and nocebo effects, we recognize the limited ability to control any psychological factors that might impact performance.

\section{Conclusion}


290

291

292

293

294

295

296

297

298

299

300

301

302

303

304

305

306

307

308

309

310

311

312

313

314

315

316

317

318

319

320

321

322

323

324

325

326

Our results demonstrate that acute ischemic preconditioning does not influence the performance of amateur soccer players during the YoYoIE2 test and does not alter the rate of perceived exertion, heart rate (peak and mean) and blood lactate among IPC, SHAM and control. However, as we have not tested the effectiveness of chronic IPC protocols (e.g., for several days or weeks), nor different IPC protocols (e.g., 3 X 3-min occlusion/2-min reperfusion) nor other types of YoYo tests (e.g., recovery level I) and mechanistic measurements (e.g., oxygen uptake, reactive oxygen species, muscle blood flow), these could be a future direction.

\section{Acknowledgments}

Financial support: The State Funding Agency of Minas Gerais, Brazil (FAPEMIG).

\section{References}

Andreas M, Schmid Al, Keilani M, Doberer D, Bartko J, Crevenna R, Moser E, and Wolzt M. 2011. Effect of ischemic preconditioning in skeletal muscle measured by functional magnetic resonance imaging and spectroscopy: a randomized crossover trial. J Cardiovasc Magn Reson 13:32. 10.1186/1532-429X-13-32

Bailey TG, Jones H, Gregson W, Atkinson G, Cable NT, and Thijssen DH. 2012. Effect of ischemic preconditioning on lactate accumulation and running performance. Med Sci Sports Exerc 44:2084-2089. 10.1249/MSS.0b013e318262cb17

Batterham AM, and Hopkins WG. 2006. Making meaningful inferences about magnitudes. Int J Sports Physiol Perform 1:50-57.

Borg E. 2007. On Perceived Exertion and its Measurement. Department of Psychology Stockholm University:58.

Borg E, and Kaijser L. 2006. A comparison between three rating scales for perceived exertion and two different work tests. Scand J Med Sci Sports 16:57-69. 10.1111/j.1600-0838.2005.00448.x

Bradley PS, Bendiksen M, Dellal A, Mohr M, Wilkie A, Datson N, Orntoft C, Zebis M, Gomez-Diaz A, Bangsbo J, and Krustrup P. 2014. The application of the Yo-Yo intermittent endurance level 2 test to elite female soccer populations. Scand J Med Sci Sports 24:43-54. 10.1111/j.16000838.2012.01483.x

Bradley PS, Di Mascio M, Bangsbo J, and Krustrup P. 2012. The maximal and sub-maximal versions of the Yo-Yo intermittent endurance test level 2 are simply reproducible, sensitive and valid. Eur J Appl Physiol 112:1973-1975. 10.1007/s00421-011-2155-1

Bradley PS, Mohr M, Bendiksen M, Randers MB, Flindt M, Barnes C, Hood P, Gomez A, Andersen JL, Di Mascio M, Bangsbo J, and Krustrup P. 2011. Sub-maximal and maximal Yo-Yo intermittent endurance test level 2: heart rate response, reproducibility and application to elite soccer. Eur J Appl Physiol 111:969-978. 10.1007/s00421-010-1721-2 
327

Caru M, Lalonde F, Gravel H, Daigle C, Tournoux F, Jacquemet V, and Curnier D. 2016. Remote ischaemic preconditioning shortens QT intervals during exercise in healthy subjects. Eur J Sport Sci 16:1005-1013. 10.1080/17461391.2016.1156161

Clevidence MW, Mowery RE, and Kushnick MR. 2012. The effects of ischemic preconditioning on aerobic and anaerobic variables associated with submaximal cycling performance. Eur J Appl Physiol 112:3649-3654. 10.1007/s00421-012-2345-5

Cruz RS, de Aguiar RA, Turnes T, Pereira KL, and Caputo F. 2015. Effects of ischemic preconditioning on maximal constant-load cycling performance. J Appl Physiol (1985) 119:961-967. 10.1152/japplphysiol.00498.2015

da Mota GR, and Marocolo M. 2016. The Effects of Ischemic Preconditioning on Human Exercise Performance: A Counterpoint. Sports Med 46:1575-1576. 10.1007/s40279-016-0595-9

Fell JW, Rayfield JM, Gulbin JP, and Gaffney PT. 1998. Evaluation of the Accusport Lactate Analyser. Int J Sports Med 19:199-204. 10.1055/s-2007-971904

Ferreira TN, Sabino-Carvalho JL, Lopes TR, Ribeiro IC, Succi JE, da Silva AC, and Silva BM. 2016. Ischemic Preconditioning and Repeated Sprint Swimming: A Placebo and Nocebo Study. Med Sci Sports Exerc. 10.1249/MSS.0000000000000977

Foster GP, WesterdahI DE, Foster LA, Hsu JV, and Anholm JD. 2011. Ischemic preconditioning of the lower extremity attenuates the normal hypoxic increase in pulmonary artery systolic pressure. Respir Physiol Neurobiol 179:248-253. 10.1016/j.resp.2011.09.001

Gibson N, Mahony B, Tracey C, Fawkner S, and Murray A. 2015. Effect of ischemic preconditioning on repeated sprint ability in team sport athletes. J Sports Sci 33:1182-1188. 10.1080/02640414.2014.988741

Gonzalez NR, Connolly M, Dusick JR, Bhakta H, and Vespa P. 2014. Phase I clinical trial for the feasibility and safety of remote ischemic conditioning for aneurysmal subarachnoid hemorrhage. Neurosurgery 75:590-598; discussion 598. 10.1227/NEU.0000000000000514

Harriss DJ, and Atkinson G. 2015. Ethical Standards in Sport and Exercise Science Research: 2016 Update. Int J Sports Med 36:1121-1124. 10.1055/s-0035-1565186

He F, Li J, Liu Z, Chuang CC, Yang W, and Zuo L. 2016. Redox Mechanism of Reactive Oxygen Species in Exercise. Front Physiol 7:486. 10.3389/fphys.2016.00486

lida H, Kurano M, Takano H, Kubota N, Morita T, Meguro K, Sato Y, Abe T, Yamazaki Y, Uno K, Takenaka $\mathrm{K}$, Hirose $\mathrm{K}$, and Nakajima T. 2007. Hemodynamic and neurohumoral responses to the restriction of femoral blood flow by KAATSU in healthy subjects. Eur J Appl Physiol 100:275-285. 10.1007/s00421-007-0430-y

Incognito AV, Burr JF, and Millar PJ. 2016. The Effects of Ischemic Preconditioning on Human Exercise Performance. Sports Med 46:531-544. 10.1007/s40279-015-0433-5

Jean-St-Michel E, Manlhiot C, Li J, Tropak M, Michelsen MM, Schmidt MR, McCrindle BW, Wells GD, and Redington AN. 2011. Remote preconditioning improves maximal performance in highly trained athletes. Med Sci Sports Exerc 43:1280-1286. 10.1249/MSS.0b013e318206845d

Krustrup P, Bradley PS, Christensen JF, Castagna C, Jackman S, Connolly L, Randers MB, Mohr M, and Bangsbo J. 2015. The Yo-Yo IE2 test: physiological response for untrained men versus trained soccer players. Med Sci Sports Exerc 47:100-108. 10.1249/MSS.0000000000000377

Laurent CM, Green JM, Bishop PA, Sjokvist J, Schumacker RE, Richardson MT, and Curtner-Smith M. 2011. A practical approach to monitoring recovery: development of a perceived recovery status scale. J Strength Cond Res 25:620-628. 10.1519/JSC.0b013e3181c69ec6

Libonati JR, Howell AK, Incanno NM, Pettee KK, and Glassberg HL. 2001. Brief Muscle Hypoperfusion/Hyperemia: An Ergogenic Aid? Journal of Strength and Conditioning Research 15:362-366. 
374

Loenneke JP, Fahs CA, Rossow LM, Sherk VD, Thiebaud RS, Abe T, Bemben DA, and Bemben MG. 2012. Effects of cuff width on arterial occlusion: implications for blood flow restricted exercise. Eur J Appl Physiol 112:2903-2912. 10.1007/s00421-011-2266-8

Marocolo M, Coriolano HA, Mourao CA, and da Mota GR. 2017. Crucial Points for Analysis of Ischemic Preconditioning in Sports and Exercise. Med Sci Sports Exerc 49:1495-1496. 10.1249/MSS.0000000000001268

Marocolo M, da Mota GR, Pelegrini V, and Appell Coriolano HJ. 2015. Are the Beneficial Effects of Ischemic Preconditioning on Performance Partly a Placebo Effect? Int J Sports Med 36:822-825. 10.1055/s-0035-1549857

Marocolo M, da Mota GR, Simim MA, and Appell Coriolano HJ. 2016a. Myths and Facts About the Effects of Ischemic Preconditioning on Performance. Int J Sports Med 37:87-96. 10.1055/s-00351564253

Marocolo M, Marocolo IC, da Mota GR, Simao R, Maior AS, and Coriolano HJ. 2016b. Beneficial Effects of Ischemic Preconditioning in Resistance Exercise Fade Over Time. Int J Sports Med 37:819-824. 10.1055/s-0042-109066

Marocolo M, Willardson JM, Marocolo IC, da Mota GR, Simao R, and Maior AS. 2016c. Ischemic Preconditioning and Placebo Intervention Improves Resistance Exercise Performance. J Strength Cond Res 30:1462-1469. 10.1519/JSC.0000000000001232

Pang CY, Yang RZ, Zhong A, Xu N, Boyd B, and Forrest CR. 1995. Acute ischaemic preconditioning protects against skeletal muscle infarction in the pig. Cardiovasc Res 29:782-788.

Patterson SD, Bezodis NE, Glaister M, and Pattison JR. 2015. The Effect of Ischemic Preconditioning on Repeated Sprint Cycling Performance. Med Sci Sports Exerc 47:1652-1658. 10.1249/MSS.0000000000000576

Paul DJ, Gabbett TJ, and Nassis GP. 2016. Agility in Team Sports: Testing, Training and Factors Affecting Performance. Sports Med 46:421-442. 10.1007/s40279-015-0428-2

Varley MC, and Aughey RJ. 2013. Acceleration profiles in elite Australian soccer. Int J Sports Med 34:3439. 10.1055/s-0032-1316315

Zhu X, and Zuo L. 2013. Characterization of oxygen radical formation mechanism at early cardiac ischemia. Cell Death Dis 4:e787. 10.1038/cddis.2013.313 


\section{Figure 1}

Figure 1

The distance covered in the YoYoIE2 test for the trials. IPC = ischemic preconditioning.

Values are mean \pm standard deviation $(n=13)$.

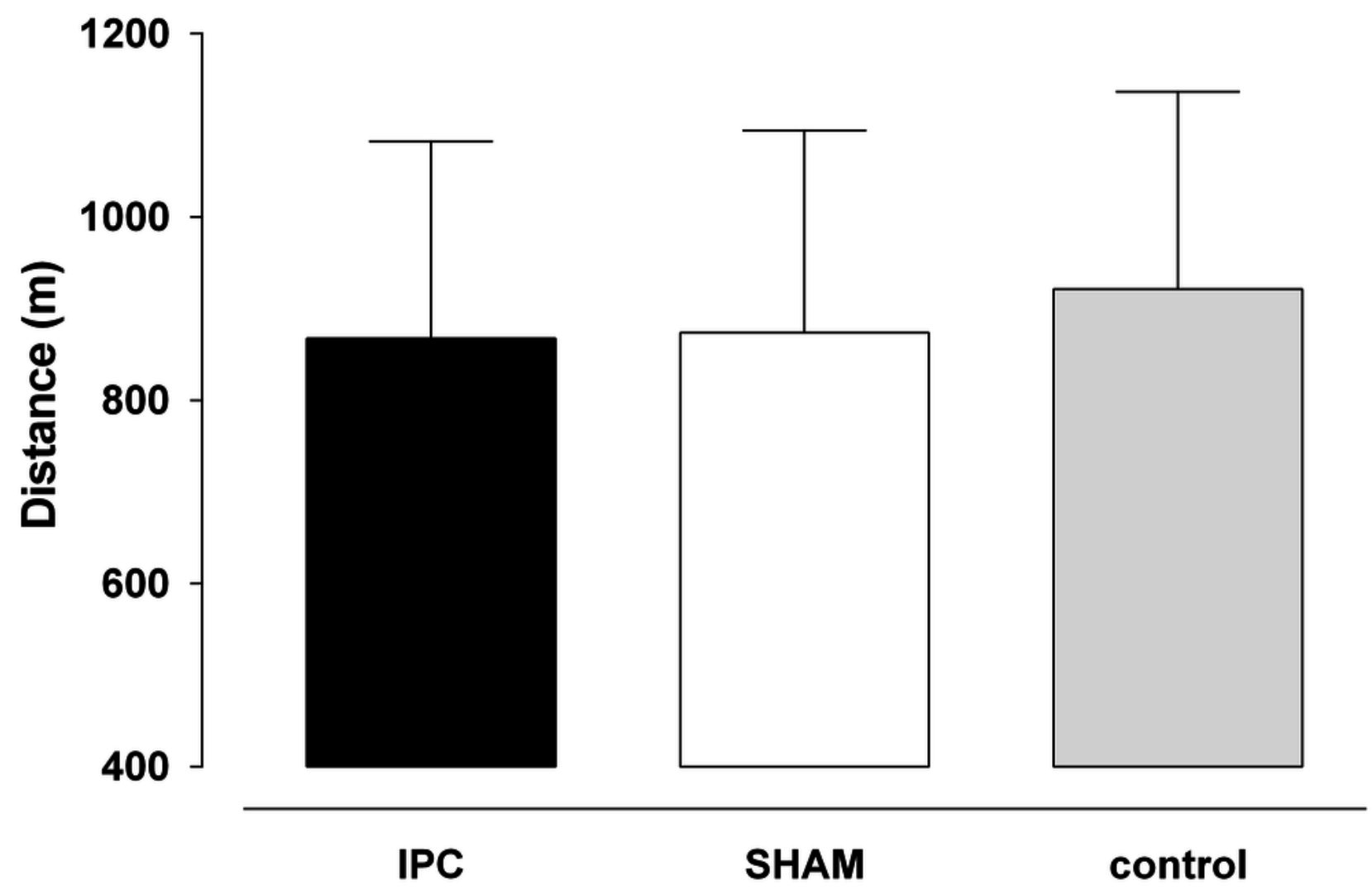




\section{Figure 2}

Figure 2

Individual changes with SHAM (A) and ischemic preconditioning - IPC (B) on the distance covered in the YoYolE2 test. Each black line represents different players ( $n=13)$. The horizontal continuous line represents the mean difference of each intervention from control. 

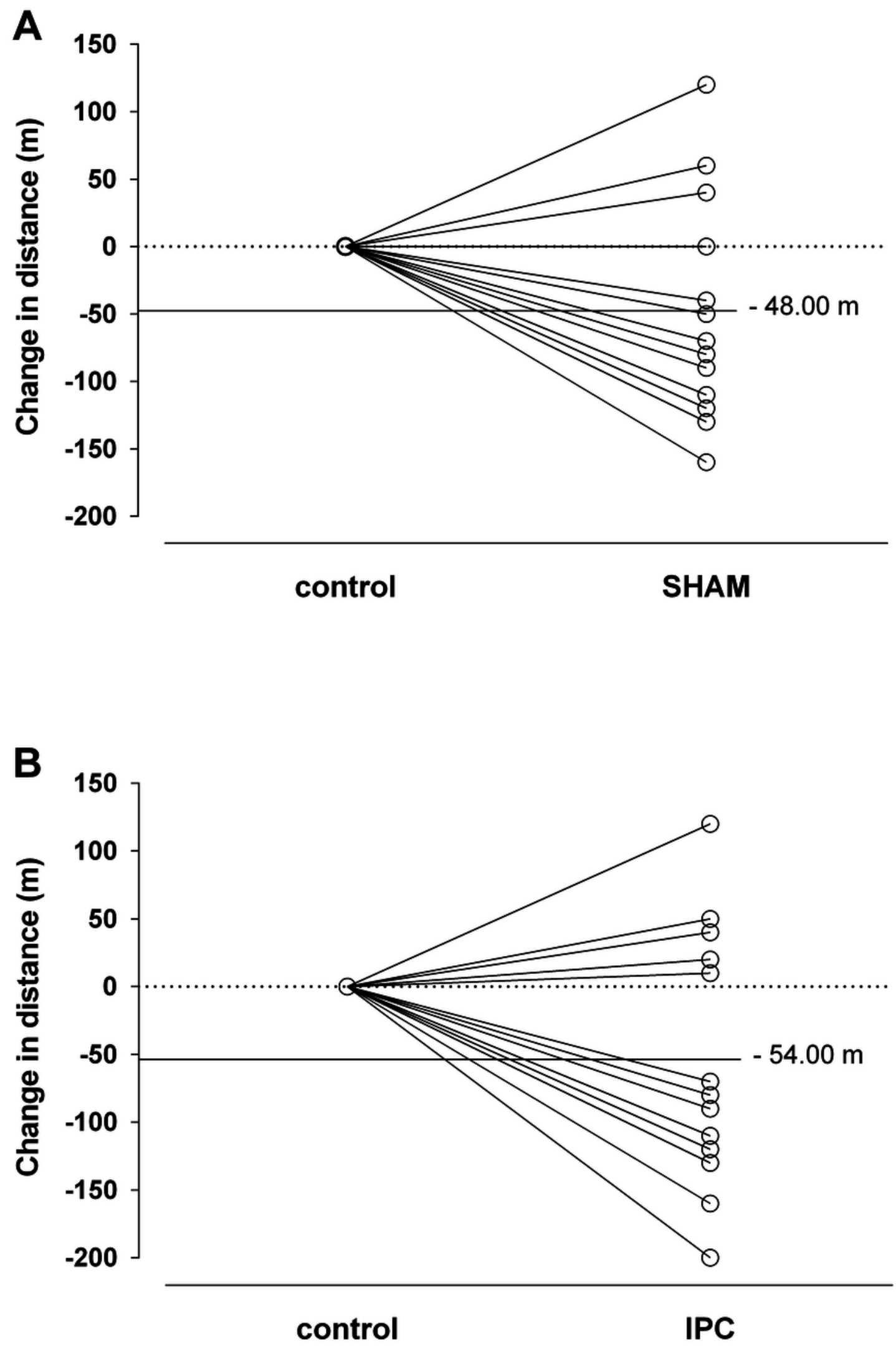


\section{Table $\mathbf{1}$ (on next page)}

Table 1: Rate of perceived exertion, blood lactate concentration, mean and peak heart rate for the trials.

Values are mean \pm standard deviation; $(n=13) ; H R=$ heart rate; IPC = ischemic preconditioning, SHAM = placebo IPC. 


\begin{tabular}{ccccc}
\hline Variable & IPC & SHAM & control & p value \\
\hline Rate of perceived exertion & $9.0 \pm 1.1$ & $8.7 \pm 1.3$ & $8.8 \pm 0.9$ & 0.69 \\
Blood lactate (mmol.L-1) & $11.1 \pm 2.7$ & $11.4 \pm 3$ & $12.7 \pm 2.4$ & 0.19 \\
HR mean (bpm) & $169 \pm 7$ & $169 \pm 5$ & $171 \pm 6$ & 0.17 \\
HR peak (bpm) & $189 \pm 5$ & $189 \pm 5$ & $191 \pm 4$ & 0.06 \\
\hline
\end{tabular}

